\title{
Review
}

Kebede W. Shinato, Feifei Huang and Ying Jin*

\section{Principle and application of atomic force microscopy (AFM) for nanoscale investigation of metal corrosion}

https://doi.org/10.1515/corrrev-2019-0113

Received December 28, 2019; accepted August 12, 2020;

published online September 15, 2020

\begin{abstract}
In this paper, the principle and application of atomic force microscopy (AFM) are reviewed and discussed in detail. Several scientific papers are used to find out data about AFM. The obtained scientific results are summarized to get a better understanding of the method and its application. The application of AFM for corrosion study is discussed in detail, and the possible conclusion is made based on the results of several articles. It is summarized that AFM is an important method to determine the surface phenomena of metal corrosion.
\end{abstract}

Keywords: alloy; atomic force microscopy; corrosion; metal.

\section{Introduction}

Atomic force microscopy (AFM), a kind of scanning probe microscopy (SPM), was invented in 1985 with the aim to measure local properties (e.g., surface topography) with a probe (Binnig et al. 1986). This method is essentially applied to investigate the characteristics of grains, phases and surface layers in a number of materials such as ceramic (Roa et al. 2011; Shuang-Yan et al. 2019), metallic (Kester et al. 2000; Qiang et al. 2011), polymer (Arseny et al. 2018; Thomé et al. 2009; Yang et al. 1995) organic or inorganic composites (Choi et al. 2007; Noel et al. 2019).

Several imaging modes are now accessible, giving an information about the sample surfaces, in addition to providing detailed three-dimensional topographies. Some

\footnotetext{
*Corresponding author: Ying Jin, National Center for Materials Service Safety, University of Science and Technology, Xueyuan 30, Beijing, 100083, PR China, E-mail: yjin@ustb.edu.cn

Kebede W. Shinato and Feifei Huang, National Center for Materials Service Safety, University of Science and Technology, Xueyuan 30, Beijing, 100083, PR China
}

of them are contact mode, tapping (or intermittent contact) and noncontact mode (Baykara and Schwarz 2017; Daniel and Nidal 2015). Contact mode is the simplest and most basic imaging mode available. During imaging the probe tip is in constant contact with the sample surface and thus operates in the repulsive regime (Daniel and Nidal 2015; Hang et al. 2018). Most commonly, the sample is imaged with a constant force maintained between the probe and the sample. Contact mode is chosen for imaging a relatively hard and smooth surface due to its relatively simple operation and accuracy in topography. Nevertheless, it has numerous limitations when imaging rough or soft surfaces, and it is powerfully influenced by tip geometry and is potentially destructive to the tip or sample surface (Daniel and Nidal 2015; Hang et al. 2018; Hilal et al. 2006). Noncontact mode is one of many vibrating cantilever techniques, where the AFM cantilever is vibrated close to the sample surface. The amplitude shift happening in the van der Waals area is used to obtain the set point. Then the amplitude set point is used as the location for the feedback loop used to regulate the sample positioning. Soft samples can be examined and specimens are not damaged or polluted through contact. This technique is only valid for use in air, where the van der Waals force governs. Even if the technique is occasionally used in liquids, it is not pretty clear how the technique really works (Antonio et al. 2006; Daniel and Nidal 2015; Hang et al. 2018; Sokolov et al. 2000). Tapping mode (also known as intermittent contact mode or amplitude modulation AFM) is a dynamic imaging method which was established to solve the characteristic drawbacks of contact mode. The cantilever is permitted to oscillate at close to its resonant frequency satisfactorily close to the sample surface such that it frequently makes contact. The interaction of the probe tip with the surface is controlled by detecting the oscillatory amplitude, which will be limited by rising contact with the sample surface. It associations abilities of both the contact and noncontact modes by collecting sample data and oscillating the cantilever tip at or close to its natural resonance frequency while letting the cantilever tip to effect the target sample for 
a slight period of time (Boussu et al. 2005; Daniel and Nidal 2015; Hang et al. 2018; Wuweikai et al. 2020).

AFM is one of the methods used to understand the corrosion characteristics of metals (Hamed et al. 2020; Lei et al. 2020; Rachid et al. 2020). Several researchers used AFM in the area of surface characterization since its development in 1980s (Baykara and Schwarz 2017; Binnig et al. 1986). Surface morphology obtained by this technique can help to examine the corrosion phenomena and the inhibition effect of corrosion inhibitors (Ambrish et al. 2020a; Shan and Frank 2019). Surface roughness can be a basic parameter to characterize the inhibition ability of corrosion inhibitors. The rougher surface is attributed to a damage on the metal surface hence the higher corrosion rate (Peter et al. 2019; Priyanka et al. 2020; Shan et al. 2020; Yakun et al. 2020). This work is a review on the principle and application of AFM for metal corrosion protection to study surface phenomena. The authors reviewed more than 50 research works in the area of AFM and metal corrosion. A conclusion has been made depending on the results of the research findings and future researched areas are suggested.

\section{Principle}

AFM is a special kind of SPM, with a verified resolution on the order nanometer (José et al. 2019; Mohammad and Arash 2020). The data is acquired by sensing the sample surface through a mechanical probe (Christopher 2019). AFM measures the force between the atoms of the probe and those of the sample (Chengwei et al. 2019; Justin et al. 2019; Taeyun et al. 2019; Zhao et al. 2019). AFM uses a cantilever with a sharp tip (probe) to scan the sample surface and measures the interaction between the sample surface and the tip for imaging or quantification of tip-sample forces (Figure 1) (Belec and Joliff 2016; Voigtländer 2019; Xiaonan et al. 2019). The cantilever is typically silicon or silicon nitride with a tip radius of on the order of nanometers. The reflected laser beam is gathered by the split photodiode. The deflections from the cantilever can be converted to numerous data sorts (Heim et al. 2004). The AFM can be used in a number of modes, based on the specific application needed by the user. Generally, the imaging modes are classified into static (contact) modes and dynamic (tapping or noncontact) modes, where the cantilever is oscillated or vibrated at a given frequency (Justin et al. 2019). Care should be given to the distance between the sample and the tip. The sample may damage due to continue force interaction between tip and sample when the tip traces the sample many times. In the dynamic mode, the cantilever vibrates at the applied frequency, as a result the lateral forces which act on the samples are intensely reduced. The dynamic mode can scan the samples more gently than the contact mode, making it appropriate for biological imaging (Ling-Zhi et al. 2019).

\section{Application of AFM in corrosion study}

Corrosion is one of the reasons for degradation and damages of the metals used in industry, transport and energy structures. The study of this phenomena and its justification is a basic issue for numerous reasons (Benaiouna et al. 2018). In order to accurately investigate the involved mechanisms, the analysis of corrosion processes needs an appropriate methods and techniques, which offer laterally resolved information, preferably in real time and under in situ corrosion conditions.

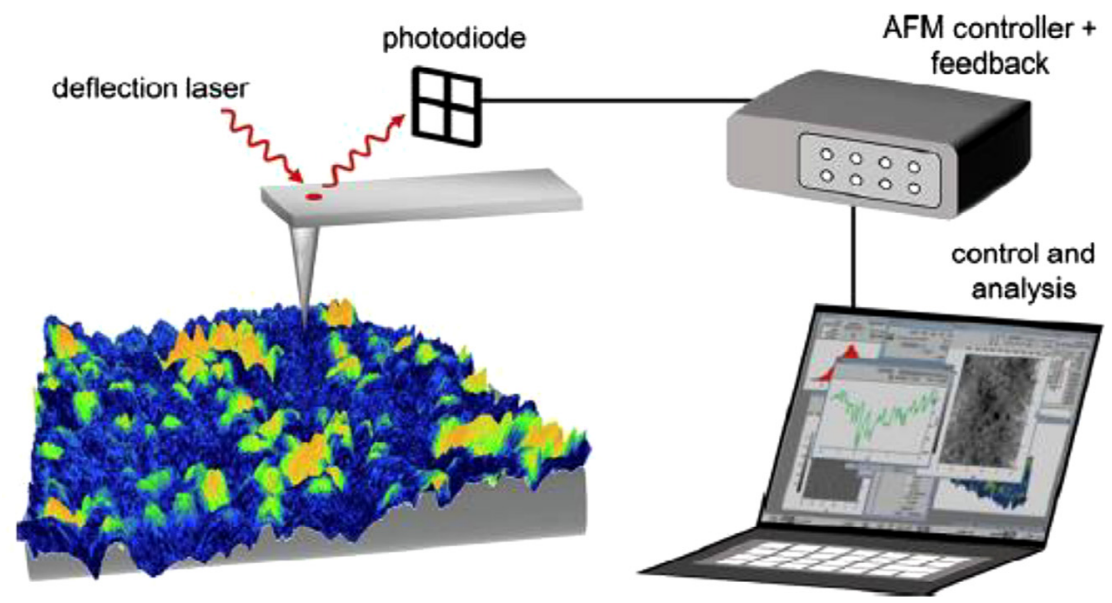

Figure 1: Schematic outlining the basic principles of atomic force microscopy (AFM). 
Typically, scanning vibrating electrode technique (SVET), electrochemical impedance spectroscopy (EIS), scanning ion-selective electrode technique, potentiodynamic polarization and AFM are employed, providing spatially resolved chemical and electrochemical information on metallic material undergoing corrosion (Ehsan et al. 2018; Izquierdo et al. 2016; Marcus and Mansfeld 2006; Oltra et al. 2007; Ying et al. 2012).

Among the techniques, AFM has been used by several researchers (in situ and/or ex situ) to describe surface phenomena of metals in different corrosive media (Tianbao et al. 2019). In order to further understand the importance of AFM instrumental technique for corrosion research, different corrosion conditions have been reviewed and explained in the following sections.

\subsection{Investigation of corrosion mechanism}

It is important to understand the corrosion mechanism of metals under corrosive media. This can help to determine how to manage the damage caused corrosion attach in a defined smart way (Ashish et al. 2020; Geringer et al. 2012; Peter et al. 2013). In this section application of AFM to investigate the corrosion mechanism of metals followed by its application on the containment of metal corrosion in different corrosion medium.

Yunzhu Shi (Yunzhu et al. 2018) studied localized corrosion of AlxCoCrFeNi high-entropy alloys in chloride solution using in situ electrochemical-AFM. Surface topography changes on the microscale/submicro scale are monitored at different applied anodizing potentials in a $3.5 \mathrm{wt} \% \mathrm{NaCl}$ solution. The microstructural evolutions with the increased $\mathrm{Al}$ content in the alloys are characterized by Scanning electron microscopy (SEM), transmission electron microscopy (TEM), energy dispersive $x$-ray spectrometry (EDS) and electron backscatter diffraction (EBSD). The results show that by increasing the Al content, the microstructure changes from single solid solution to multiphases, leading to the segregations of elements and it is clearly observed by AFM morphological result as shown in Figure 2.

Davoodi et al. on the other hand used Integrated AFM and SECM for in situ studies of localized corrosion of $\mathrm{Al}$ alloys (Davoodi et al. 2007). Their study aimed at exploring the differences in intrinsic corrosion properties between EN AW-3003 and a newly developed Al-Mn-Si-Zr fin alloy. Volta potential mapping of both alloys by scanning Kelvin probe force microscopy (SKPFM) specifies a cathodic behavior of constituent intermetallic particles $(>0.5 \mathrm{~m})$
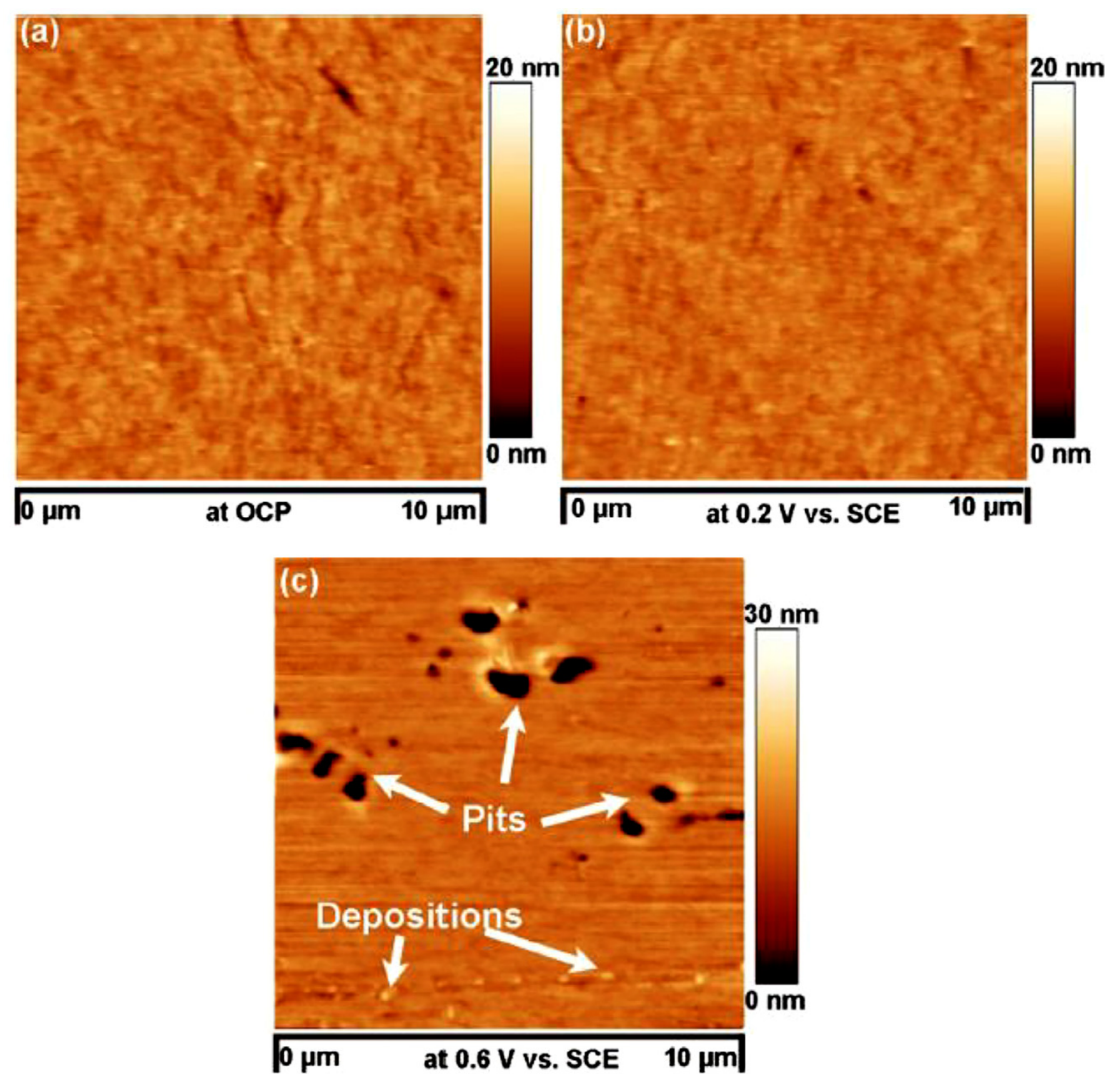

Figure 2: In situ EC-atomic force microscopy (AFM) topography images of the Al0.3CoCrFeNi HEA in a $3.5 \mathrm{wt} \% \mathrm{NaCl}$ solution after 10-min exposure at (a) OCP, (b) $0.2 \mathrm{~V}$, and (c) $0.6 \mathrm{~V}$ versus SCE, respectively. 
relative to the alloy matrix. Compared to EN AW-3003, the Al-Mn-Si-Zr alloy has a smaller number of particles with large Volta potential difference relative to the matrix (Figure 3). In situ AFM measurements in slightly corrosive solutions showed extensive localized dissolution and deposition of corrosion products on EN AW-3003 and only a small number of corroding sites and "tunnel-like" pits on Al-Mn-Si-Zr. Probing the ongoing localized corrosion process by integrated AFM and scanning electrochemical microscopy (SECM) revealed more extensive local electrochemical activity on EN AW-3003 than on Al-Mn-SiZr. This shows AFM has a great contribution to study surface phenomena of localized corrosion.

Surface potential of particles on the surface metals can be measured by AFM scanning potential technique. Which gives information about corrosion resistance ability of a given metal in a particular corrosive medium (Birgul and Cansu 2017; Schmutz and Frankel 1999). Campestrini et al. studied Relation between microstructural aspects of AA2024 and its corrosion behavior using AFM scanning potential technique. The results of which shows that formation of shell-shaped particles makes the aluminum alloy more susceptible to the pitting attack. a large difference in potential between the core and the shell, which results in a highly localized galvanic coupling. Therefore, the development of shell-shaped particles principally drops the resistance to pitting attack of the AA2024 (Campestrini et al. 2000).

Researchers in atmospheric corrosion also used AFM techniques. Tingru et al. studied atmospheric corrosion of copper and Cu5Zn5Al1Sn alloy (Tingru et al. 2018). They used AFM as one technique to understand the atmospheric corrosion of the specified substrates and coppered each other. The heterogeneities in Volta potential are often regarded as variations in relative nobility along the surface which may trigger micro-galvanic corrosion effects. An unexposed Cu5Zn5Al1Sn surface where scratches clearly are seen after the diamond polishing (Figure 4a). Corresponding volta potential map (Figure 4b) showing no significant variations along the investigated surface area. This lack of variations is in contrast to corresponding SKPFM studies of a diamond polished single-phase Cu20Zn alloy in which variations in Volta potential between different grains could be discerned, most likely due to variations in Zn-content between individual grains.

\subsection{Corrosion inhibitor evaluation}

A corrosion inhibitor is a substance when added in a small concentration to an environment that reduces the corrosion rate of a metal exposed to a corrosive environment. Inhibitors often play an important role in the oil extraction and processing industries, where they have always been considered to be the first line of defense against corrosion (Ansari et al. 2020; Heloisa et al. 2015; Obi-Egbedi 2010). The use of corrosion inhibitors is the most economical and convenient technique to control a corrosive attack on metals. The importance of a corrosion study depends on the fact that corrosion causes great losses to the economy
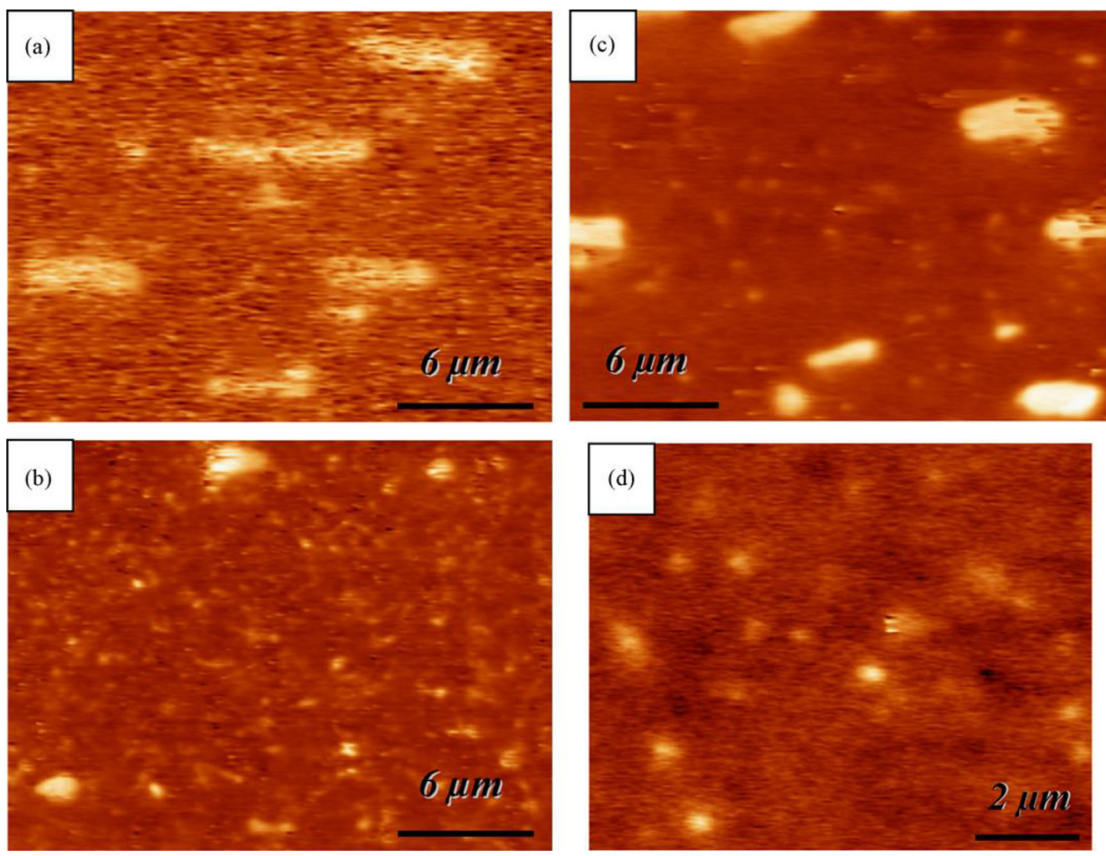

Figure 3: Volta potential maps of polished (a and b) EN AW-3003 and (c and d) Al-Mn-Si$\mathrm{Zr}$, showing higher Volta potential (brighter) of larger micrometer sized particles ( $a$ and c) and smaller particles of a few hundred nanometer in size (b and d). 

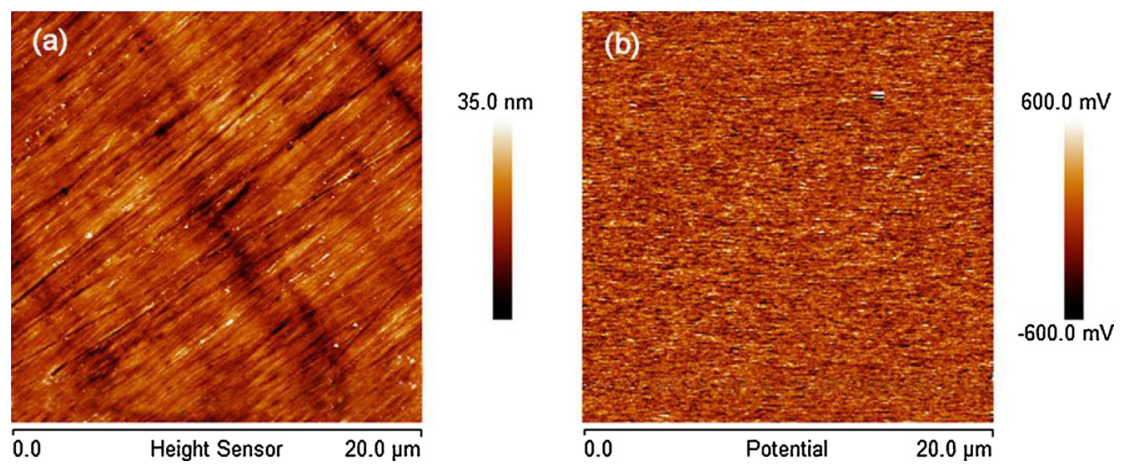

Figure 4: Atomic force microscopy (AFM)based topography (a) and Volta potential variations (b) obtained with scanning Kelvin probe force microscopy (SKPFM) of unexposed freshly diamond polished Cu5Zn5Al1Sn.

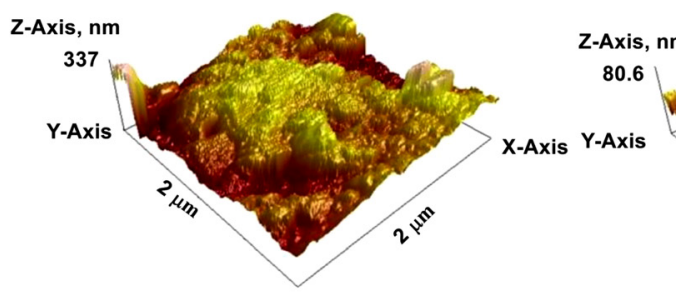

(a)

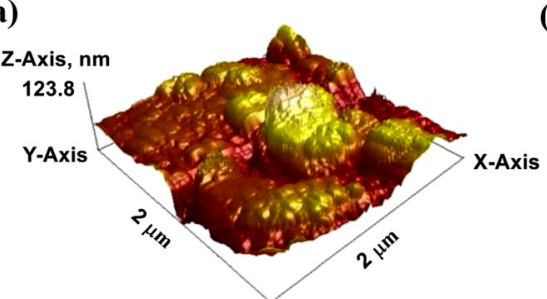

(c)

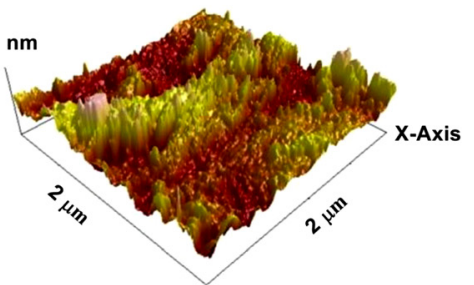

(b)

Figure 5: Three-dimension atomic force microscopy (AFM) images: (a) without CPs, (b) with CP-1 and (c) with CP-2.

and is a major threat to human safety (Ben et al. 2020; Emetere and Olawole 2019; Shalabi and Ahmed 2019). A number of synthetic compounds are known to be applicable as good corrosion inhibitors for metals.

Several methods are used to evaluate the protection capability of corrosion inhibitors (Hoomaan et al. 2020; Ismail 2007; Karthik and Sundara 2016; Li et al. 2014; Tan et al. 2017). Some of the most widely used methods are weight loss measurement, potentiodynamic polarization measurement, EIS, SECM and SVET, X-ray photoelectron spectroscopy and AFM. Many methods can be used to solid research output and each of the above have their own advantage and limitation. Since our topic is about AFM, let us continue some evidence how it can be applicable to investigate corrosion inhibition ability of inhibitors.

Singh et al. used pyrimidine derivatives as green and sustainable corrosion inhibitor for N80 steel in highly corrosive environment. For their work they synthesize two chromenopyrimidine derivatives (CPs) namely 8,8-Dimethyl-5-p-tolyl-8,9-dihydro-1H-chromeno
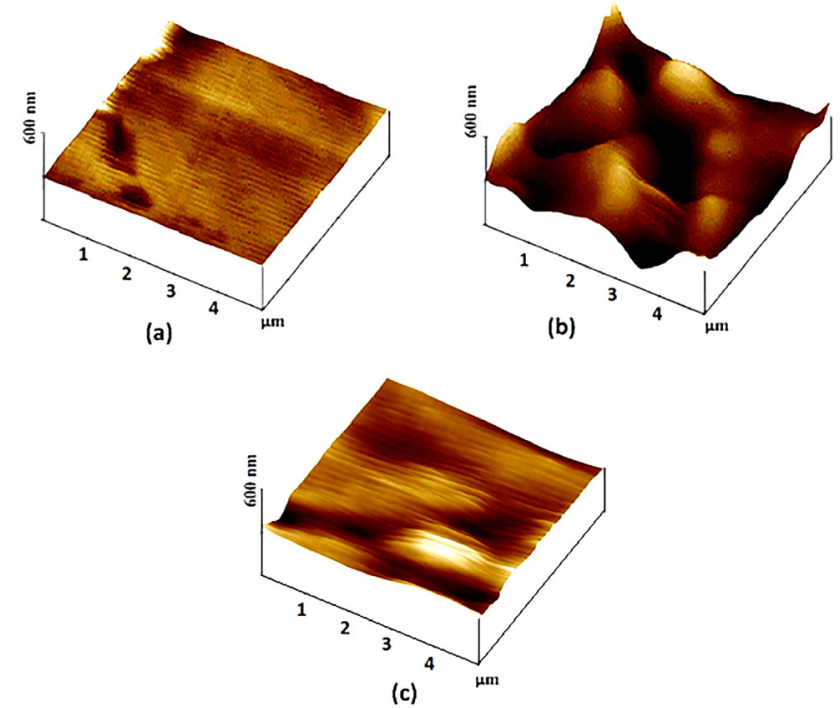

Figure 6: 3D images of the copper surface: (a) before and (b) after 1-h soaking time in the blank and (c) after 1-h soaking time in the $1 \mathrm{mM}$ PyTA/1 $\mathrm{M} \mathrm{HCl}$. 
pyrimidine-2,4,6 (3H, 5H, 7H)-trione (CP-1) and 8,8Dimethyl-5-phenyl- 8,9-dihydro-1H-chromeno pyrimidine2,4,6 $(3 \mathrm{H}, 5 \mathrm{H}, 7 \mathrm{H})$-trione $(\mathrm{CP}-2)$ and characterized their inhibition effect for N80 steel using several methods (Ambrish et al. 2020b). Electrochemical techniques were used to calculate the inhibition efficiency, AFM on the other hand for surface morphology characterization. The AFM surface morphology of unexposed N80 steel, exposed without and with inhibitor samples were taken and compared as shown in Figure 5. From the result, the N80 steel surface greatly damaged by the corrosive attack of the 15\% $\mathrm{HCl}$ (Figure 5a) and the peaks and valleys around $337 \mathrm{~nm}$. However, the addition of inhibitor in the corrosive medium minimizes the negative impact of the corrosive medium, which is evidently seen by the 3D images (Figure 5b, c) with smooth surface morphology. Likewise, Razieh Farahati et al. used AFM to determine the corrosion inhibitive effect of 4-(pyridin-3-yl) thiazol-2-amine for copper in chloride-containing solution. A smoot surface morphology image obtained by AFM investigation (Figure 6) clearly used to explain the inhibition ability the specified inhibitor for copper. It is worth to mention research papers which use AFM as a tool for corrosion inhibition evaluation. The surface morphology and surface roughness obtained by AFM test is a key point to understand the inhibitive action of corrosion inhibitor for different metals (Bochuan et al. 2019; Ting et al. 2020).
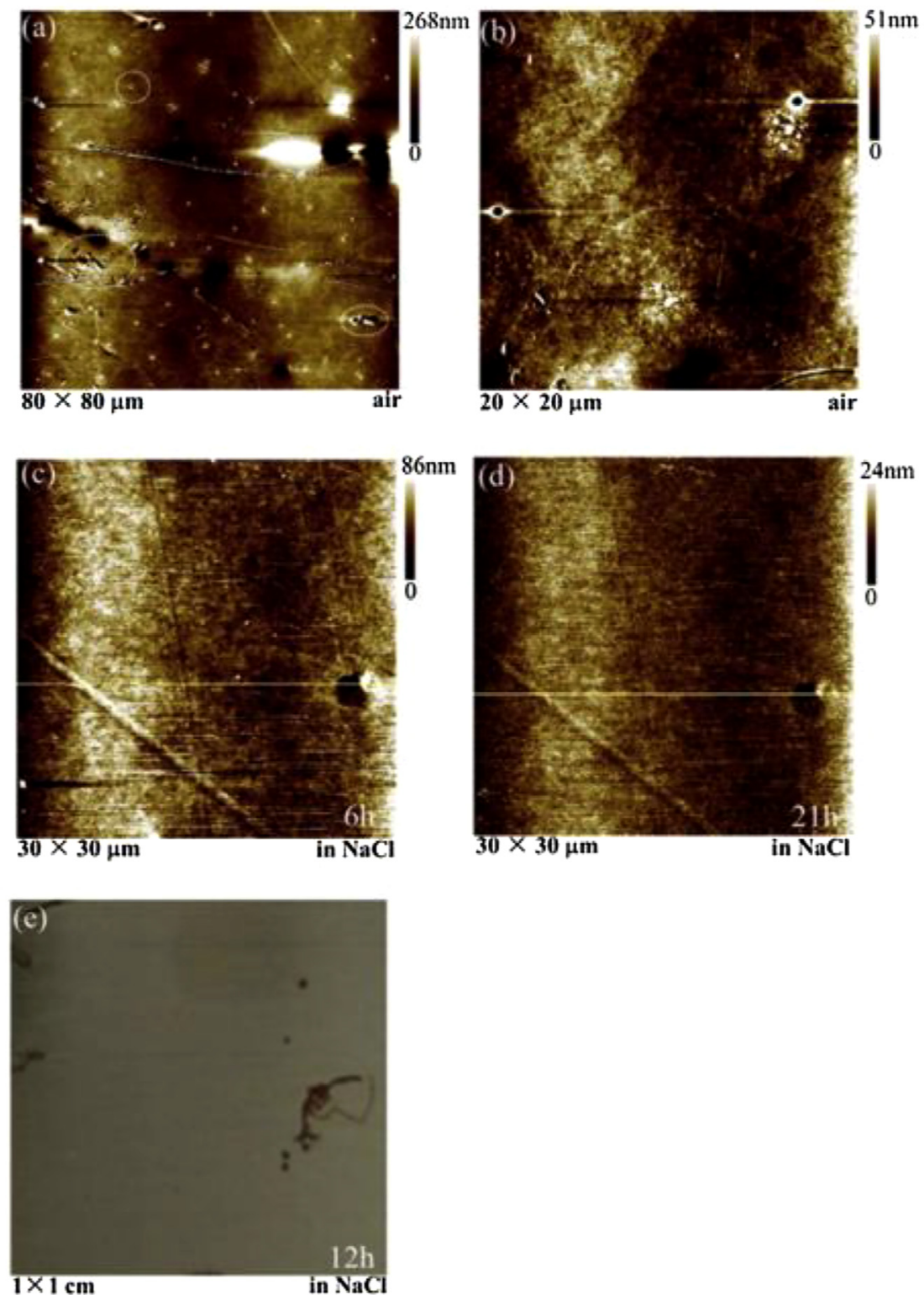

Figure 7: In situ and/or ex situ atomic force microscopy (AFM) images of the alkyd coating obtained in air (a,b) and in 3 wt $\%$ $\mathrm{NaCl}$ solution (c, d), respectively. A photo (e) of the alkyd coating was taken after $12 \mathrm{~h}$ exposure. 
Table 1: Roughness, pinhole diameter/depth and Z-scale values of the alkyd coating.

\begin{tabular}{|c|c|c|c|c|c|c|}
\hline Alkyd coating & Scan size $(\mu \mathrm{m})$ & Time (h) & $\mathrm{Ra}(\mathrm{nm})$ & $\mathrm{Rq}(\mathrm{nm})$ & $\begin{array}{r}\text { Diameter }(\mathrm{nm}) / \\
\text { depth of the pinhole }(\mathrm{nm})\end{array}$ & Z-Scale (nm) \\
\hline \multirow[t]{3}{*}{ In air } & $80 \times 80$ & - & 19 & 23 & - & 268 \\
\hline & $30 \times 30$ & - & 3 & 4 & - & 66 \\
\hline & $20 \times 20$ & - & 1 & 1 & - & 51 \\
\hline \multirow[t]{2}{*}{ In $\mathrm{NaCl}$ solution } & $30 \times 30$ & 6 & 5 & 6 & $1.3 / 8.5$ & 86 \\
\hline & $30 \times 30$ & 12 & 3 & 4 & $1.1 / 7.8$ & 24 \\
\hline
\end{tabular}

\subsection{Coating performance evaluation}

Coating is another approach to prevent metals from corrosion by incorporating a corrosion resistant layer at the top of the substrate. The materials important for coating first synthesized then added to the target substrate and evaluate its corrosion performance. In this process, AFM can be used to characterize the corrosion performance of a coated material on the metal surface (Mei et al. 2016). Jing et al. used solvent borne alkyd coating with nanoclay for corrosion protection of carbon steel. For this study they used in situ AFM as surface characterization tool (Jing et al. 2015). They find out that the surface morphology of blank metal surface is different with that of coated one and made a conclusion according to the result obtained by this method. As shown in Figure 7a, in addition to hills and valleys, there are defects such as pinholes and microcracks in the coating (marked by dot circles), and Figure $7 \mathrm{~b}$ shows more clearly the pinholes. The pinholes and microcracks can become easy pathways for penetration of water and corrosive ions. The coating surface is relative smooth, with a roughness value ca. $20 \mathrm{~nm}$ on an area of $80 \mathrm{~m}$ in size. The sequential in situ AFM images in Figure 7c, d shows that, the coating surface changed significantly during $21 \mathrm{~h}$ immersion in the $\mathrm{NaCl}$ solution, the diameter and depth of the shallow pinhole, the roughness and Z-scale of the coating surface decreased with the exposure time (Table 1), implying a low stability of the coating. The photo of the alkyd coating (Figure 7e) taken after $12 \mathrm{~h}$ exposure shows some brownish corrosion products, most likely red rust formed due to a fast transport of the electrolyte through the coating. These in situ AFM results provide evidences that

Table 2: Advantages and disadvantages of AFM.

\section{No. Advantages}

1. AFM can be undertaken in a number of different environments, encompassing ambient, liquid and vacuum. It is ideally suited to the analysis of biological samples due to its ability to image in a liquid environment.

2. SEM can only be carried out with samples that are conductive. As a result, a nonconductive sample must be covered in metal to allow imaging to take place. That would irreversibly change or damage the sample. This additional work to prepare the sample is not necessary with AFM.

3. AFM can produce a three-dimensional image of a sample surface with quantification of the surface roughness, while only two dimensions of imaging are possible with SEM.

4. Compared with 2D TEM, 3D AFM images are obtained without expensive sample preparation and yield more information.

5. Scanning electrochemical microscopy (SECM) is an electroanalytical and unique scanning probe technique which can image substrate topography and local reactivity with high resolution.
Disadvantages

AFM can only image a maximum height on the order of 10-20 $\mu \mathrm{m}$ and a maximum scanning area of about $150 \times 150 \mu \mathrm{m}$. In one pass, the SEM can image an area on the order of square millimeters with a depth of field on the order of millimeters.

The scanning speed of an AFM is also a limitation. Traditionally, an AFM cannot scan images as fast as a SEM, requiring several minutes for a typical scan, while a SEM is capable of scanning at near real-time, although at relatively low quality.

As with any other imaging technique, there is the possibility of image artifacts, which could be induced by an unsuitable tip, a poor operating environment, or even by the sample itself. These image artifacts are unavoidable; however, their occurrence and effect on results can be reduced through various methods.

TEM is a super-resolution imaging technique that produces a $2 \mathrm{D}$ image using electrons instead of photons. TEM offers valuable information on the inner structure of the sample, such as crystal structure, morphology and stress state information.

One of the major downsides is the single scan image size, which is of the order of $150 \times 150 \mu \mathrm{m}$, compared with millimeters for a scanning electron microscope. 
the intercalated modified montmorillonite (MMT) nanoclay in the alkyd matrix can reduce the water penetration and thus postpone the onset of corrosion reaction.

\section{Advantage and disadvantage of AFM}

Like other instrumental methods, AFM has its own advantage and disadvantage over other relatively similar functioning instruments like scanning electron microscopy and summarized in Table 2 . When determining whether or not analyzing a sample with an AFM is appropriate, this advantages and disadvantages that must be taken into account to get a better experimental result. From Table 2 we can observe that AFM has its own advantage and disadvantage over SEM, TEM and SECM. Its functionality in a number of different environments, encompassing ambient, liquid, and vacuum makes AFM more applicable than SEM. On the other hand, AFM is versatile because it can not only image in 3D topography but also provide various types of surface measurements this gives an advantage over TEM instrumentation, which produces a 2D image.

\section{Summary and conclusions}

In this review paper, the principle and application of AFM are described. It is a special kind of SPM, with a verified resolution on the order of nanometer. AFM can be classified into contact and on-contact or static and dynamic depending on its application. Based on the reviewed research findings, it has several applications starting from biological application to metal protection. AFM is an effective method to contribute to the understanding of corrosion and corrosion inhibition mechanisms of metals both qualitatively and quantitatively. AFM has demonstrated several applications in corrosion studies, including corrosion inhibition evaluation, coating performance evaluation and atmospheric corrosion studies.

Author contribution: All the authors have accepted responsibility for the entire content of this submitted manuscript and approved submission.

Research funding: None declared.

Conflict of interest statement: The authors declare no conflicts of interest regarding this article.

\section{References}

Ambrish, S., Ansari, K.R., Dheeraj, S.C., Quraishi, M.A., Lgaz, H., and Ill-Min, C. (2020a). Comprehensive investigation of steel corrosion inhibition at macro/ micro level by ecofriendly green corrosion inhibitor in $15 \% \mathrm{HCl}$ medium. J. Colloid Interface Sci. 560: 225-236.

Ambrish, S., Ansari, K.R., Dheeraj, S.C., Quraishi, M.A., and Savas, K. (2020b). Anti-corrosion investigation of pyrimidine derivatives as green and sustainable corrosion inhibitor for N80 steel in highly corrosive environment: experimental and AFM/XPS study. Sustain. Chem. Pharm. 16: 100257.

Ansari, K.R., Chauhan, D.S., and Quraishi, M.A. (2020). Chitosan Schiff base: an environmentally benign biological macromolecule as a new corrosion inhibitor for oil \& gas industries. Int. J. Biol. Macromol. 144: 305-315.

Antonio, C., Yuguang, C., Oleg, G., and Benjamin, M.O. (2006). High resolution non-contact AFM imaging of liquids condensed onto chemically nanopatterned surfaces. Ultramicroscopy 106: 703708.

Arseny, K., Valentin, A., Oleg, P., Andrei, L.K., and Alexander, T. (2018). An atomic force microscopy mode for nondestructive electromechanical studies and its application to diphenylalanine peptide nanotubes. Ultramicroscopy 185: 49-54.

Ashish, K.S., Bhawna, C., Sanjeeve, T., Balaram, P., Hassane, L., IllMin, C., Shweta, P., and Rajiv, P. (2020). Green approach of synthesis of thiazolyl imines and their impeding behavior against corrosion of mild steel in acid medium. Colloids Surf. A 599: 124824.

Baykara, M.Z. and Schwarz, U.D. (2017). Atomic force microscopy: methods and applications, encyclopedia of spectroscopy and spectrometry, 3rd ed: Elsevier, pp. 70-75.

Belec, L. and Joliff, Y. (2016). Mechanically affected zone in AFM force measurements - focus on actual probe tip geometry. Mater Design 104: 217-226.

Ben, M., Abubshait, S., and Etteyeb, N. (2020). Olive leaf extract as a green corrosion inhibitor of reinforced concrete contaminated with seawater. Ara. J. Chem. 13: 4846-4856.

Benaiouna, N.E., Maafa, I., Florentin, A., Denys, E., Hakiki, N.E., Moulayat, N., and Bubendorff, J.L. (2018). Time dependence of the natural passivation process on AISI 304 in an alkaline medium: atomic force microscopy and scanning Kelvin probe force microscopy as additional tools to electrochemical impedance spectroscopy. Appl. Surf. Sci. 436: 646-652.

Binnig, G., Quate, C.F., and Gerber, C. (1986). Atomic force microscope. Phys. Rev. Lett. 56: 930-933.

Birgul, B., and Cansu, Y. (2017). The influence of silver and copper ions on the antibacterial activity and local electrical properties of single sepiolite fiber: a conductive atomic force microscopy (C-AFM) study. Appl. Clay Sci. 146: 449-456.

Bochuan, T., Shengtao, Z., Hongyan, L., Yuwan, G., Yujie, Q., Wenpo, L., Lei, G., Chunliu, X., and Shijin, C. (2019). Corrosion inhibition of $\mathrm{X} 65$ steel in sulfuric acid by two food flavorants 2-isobutylthiazole and 1-(1,3-Thiazol-2-yl) ethanone as the green environmental corrosion inhibitors: combination of experimental and theoretical researches. J. Colloid Interface Sci. 538: 519-529.

Boussu, K., Bruggen, V., Volodin, A., Snauwaert, J., Haesendonck, C., and Vandecasteele, C. (2005). Roughness and hydrophobicity studies of nanofiltration membranes using different modes of AFM. J. Colloid Interface Sci. 286: 632-638.

Campestrini, P., Westinga, E.P.M., Rooijena, H.W., and Wit, J.H.W. (2000). Relation between microstructural aspects of AA2024 and 
its corrosion behaviour investigated using AFM scanning potential technique. Corros. Sci. 42: 1853-1861.

Chengwei, X., Liping, L., and Ming, W. (2019). A new preparation method and imaging parameters of asphalt binder samples for atomic force microscopy. Construct. Build. Mater. 205: 622632.

Choi, D., Jeon, J., Lee, P., Hwang, W., Lee, K., and Park, H. (2007). Young's modulus measurements of nanohoneycomb structures by flexural testing in atomic force microscopy. Compos. Struct. 79: 548-553.

Christopher, J.R. (2019). Conductive atomic force microscopy data from substantia nigra tissue. Data in brief 27: 103986.

Daniel, J. and Nidal, H. (2015). Characterisation and quantification of membrane surface properties using atomic force microscopy: a comprehensive review. Desalination 356: 149-164.

Davoodi, A., Pan, J., Leygraf, C., and Norgren, S. (2007). Integrated AFM and SECM for in situ studies of localized corrosion of Al alloys. Electrochim. Acta 52: 7697-7705.

Ehsan, R., Ali, R.A., Alireza, K.R., Hamidreza, J., and Ali, D. (2018). Morphology modification of electrodeposited superhydrophobic nickel coating for enhanced corrosion performance studied by AFM, SEM-EDS and electrochemical measurements. Colloids Surf. A 547: 81-94.

Emetere, M.E. and Olawole, O.C. (2019). Environmental effect of corrosion on meteorological parameters over malabo over malabo. Procedia Manuf. 35: 854-860.

Geringer, J., Pellier, J., Cleymand, F., and Forest, B. (2012). Atomic force microscopy investigations on pits and debris related to frettingcorrosion between 316LSS and PMMA. Wear 292: 207-217.

Hamed, P., Mehdi, S.N., Mohammad, R.G., and Mohammad, H.O. (2020). Inhibition of acid corrosion of glass ampoule in $\mathrm{Pb} / \mathrm{HBF}_{4} /$ $\mathrm{PbO}_{2}$ reservebatteries using nanobis [3-(trimethoxysilyl)propyl] amine. J. Mol. Liq. 302: 112578.

Hang, Z., Junxiang, H., Yongwei, W., Rui, L., Xiulan, H., Jingjing, J., and Chantelle, A. (2018). Atomic force microscopy for two-dimensional materials: a tutorial review. Optic Commun. 406: 3-17.

Heim, T., Lmimouni, K., and Vuillaume, D. (2004). Ambipolar charge injection and transport in a single pentacene monolayer island. Nano Lett. 4: 2145-2150.

Heloisa, P.D., Pedro, V.D., Luiz, C.P.A., Gabriela, V., Eustáquio, V.R.C., Glória, M.F.A., Alexandre, O.G., Robson, R.M., Valdemar, L., Boniek, G.V., et al. (2015). Evidencing the crude oil corrosion by Raman spectroscopy, atomic force microscopy and electrospray ionization FT-ICR mass spectrometry. Fuel 139: 328-336.

Hilal, N., Bowen, W.R., Alkhatib, L., and Ogunbiyi, O. (2006). Areview of atomic force microscopy applied to cell interactions with membranes. Chem. Eng. Res. Des. 84: 282-292.

Hoomaan, J.M., Ali, M., Seid, M.J., Mehrasa, A., Mohsen, S., and Amir, B. (2020). Fabrication and characterization of graphene oxidechitosan-zinc oxide ternary nano-hybrids for the corrosion inhibition of mild steel. Int. J. Biol. Macromol. 148: 1190-1200.

Ismail, K.M. (2007). Evaluation of cysteine as environmentally friendly corrosion inhibitor for copper in neutral and acidic chloride solutions. Electrochim. Acta 52: 7811-7819.

Izquierdoa, J., Fernández-Pérezb, B.M., Eiferta, A., Souto, R.M., and Kranza, C. (2016). Simultaneous atomic force-scanning electrochemical microscopy (afm-secm) imaging of copper dissolution. Electrochim. Acta 201: 320-332.

Jing, L., Luiz, E., Michele, F., Valentina, E., Gregory, D., and Jinshan, P. (2015). In-situ AFM and EIS study of a solventborne alkyd coating with nanoclay for corrosion protection of carbon steel. Prog. Org. Coating 87: 179-188.

José, J.B., Susana, G.P., Eva, D., Antonio, H., and José, A.H.G. (2019). Applications and potentialities of Atomic Force Microscopy in fossil and extant plant cuticle characterization. Rev. Palaeobot. Palynol. 268: 125-132.

Justin, G., Lal, B.A., Anesh, M.P., Yiran, A., and Ajit, K.S. (2019). Nanomechanical behaviour of biochar-starch polymer composite: investigation through advanced dynamic atomic force microscopy. Composites Part A 124: 105486.

Karthik, G. and Sundara, V.M. (2016). Investigations of the inhibition of copper corrosion in nitric acid solutions by levetiracetam drug. Egypt. J. Pet. 25: 481-493.

Kester, E., Rabe, U., Presmanes, L., Tailhades, P., and Arnold, W. (2000). Measurement of Young's modulus of nanocrystalline ferrites with spinel structures by atomic force acoustic microscopy. J. Phys. Chem. Solid. 61: 1275-1284.

Lei, G., Jianhong, T., Savas, K., Senlin, L., Qingbiao, L., and Fan, Z. (2020). Multidimensional insights into the corrosion inhibition of 3,3- dithiodipropionic acid on Q235 steel in $\mathrm{H}_{2} \mathrm{SO}_{4}$ medium: a combined experimental and in silico investigation. J. Colloid Interface Sci. 570: 116-124.

Li, C., Li, L., and Wang, C. (2014). Study of the inhibitive effect of mixed self-assembled monolayers on copper with SECM. Electrochim. Acta 115: 531-536.

Ling-Zhi, C., Weidong, Z., Shuang, S., and Cai, S. (2019). Lab on a tip: applications of functional atomic force microscopy for the study of electrical properties in biology. Acta Biomater. 99: 33-52.

Marcus, P. and Mansfeld, F. (2006). Analytical methods in corrosion science and engineering. Boca Raton (FL, USA): CRC Press.

Mei, Y., Min, L., Jianhua, L., Songmei, L., Bing, X., and Hao, Z. (2016). Effect of chelating agent acetylacetone on corrosion protection properties of silane-zirconium sol-gel coatings. Appl. Surf. Sci. 363: 229-239.

Mohammad, S.M. and Arash, B. (2020). A novel excitation scheme to enhance image resolution in dynamic atomic force microscopy. Phys. Lett. 384: 126099.

Noel, B., Juan, M.M., Israel, A.V., Georgina, C.D., and Pablo, D.R.G. (2019). Nano- and micro-mechanical properties of wheat grain by atomic force microscopy (AFM) and nano-indentation (IIT) and their relationship with the mechanical properties evaluated by uniaxial compression test. J. Cereal. Sci. 90: 102830.

Obi-Egbedi, I. (2010). An interesting and efficient green corrosion inhibitor for aluminium from extracts of Chlomolaena odorata in acidic solution. J. Appl. Electrochem. 40: 1977-1984.

Oltra, R., Maurice, V., Akid, R., and Marcus, P. (2007). Local probe techniques for corrosion research. Cambridge (England): Woodhead Publishing.

Peter, G., Assya, B., Bistra, K., Denitsa, M., Thomas, B., and Konstantin, B. (2013). Implementing atomic force microscopy (AFM) for studying kinetics of gold nanoparticle's growth. Colloid. Surface. Physicochem. Eng. Aspect. 434: 154-163.

Peter, R., Ingrid, M., Maria, L., Francesco, A., and Lorenzo, F. (2019). Study of the synergistic effect of cerium acetate and sodium sulphate on the corrosion inhibition of AA2024-T3. Electrochim. Acta 308: 337349.

Priyanka, S., Chauhan, D.S., Chauhan, S.S., Singh, G., and Quraishi, M.A. (2020). Bioinspired synergistic formulation from dihydropyrimdinones and iodide ions for corrosion inhibition of carbon steel in sulphuric acid. J. Mol. Liq. 298: 112051. 
Qiang Li, G.Z., Adam, S.Z., Yi, L., Krystyn, J., Van, V., Carl, V., and Thompson, C.V. (2011). Compositional dependence of Young's moduli for amorphous Cu-Zr films measured using combinatorial deposition on microscale cantilever arrays. Scripta Mater. 64: 41-44.

Rachid, H., Fouad, B., Omar, D., Mehdi, E.B., Khalid, N., Mohammed, A., Samir, B., Abdelkader, Z., and Ahmed, E. (2020). Development and potential performance of prepolymer in corrosion inhibition for carbon steel in $1.0 \mathrm{M} \mathrm{HCl}$ : outlooks from experimental and computational investigations. J. Colloid Interface Sci. 574: 43-60.

Roa, J.J., Oncins, G., Dias, F.T., Vieira, V.N., Schaf, J., and Segarra, M. (2011). AFM as an alternative for Young's modulus determination in ceramic materials in elastic deformation regime. Phys. $C$ Supercond. 471: 544-548.

Schmutz, P. and Frankel, G.S. (1999). Influence of dichromate ions on corrosion of pure aluminum and AA2024-T3 in NaCl solution studied by AFM scratching. J. Electrochem. Soc. 146: 12 .

Shalabi, K. and Ahmed, A.N. (2019). Ethoxylates nonionic surfactants as promising environmentally safe inhibitors for corrosion protection of reinforcing steel in $3.5 \% \mathrm{NaCl}$ saturated with $\mathrm{Ca}(\mathrm{OH})_{2}$ solution. J. Mol. Struct. 1195: 863-876.

Shan, Q. and Frank, Y.C. (2019). Synergism of imidazoline and sodium dodecylbenzenesulphonate inhibitors on corrosion inhibition of X52 carbon steel in $\mathrm{CO}_{2}$-saturated chloride solutions. J. Mol. Liq. 294: 111674.

Shan, W., Xiao-Ze, M., Chun-Hui, M., Xin-Xin, Z., and Ze-Hua, D. (2020). Inhibition of 2-phenyl imidazoline on chloride-induced initial atmospheric corrosion of copper by quartz crystal microbalance and electrochemical impedance. Corros. Sci. 170: 108692.

Shuang-Yan, L., Yang, S., Xin-Cheng, H., Hui-Juan, Y., Rui, W., and Wan, L.-J. (2019). Recent progress in the application of in situ atomic force microscopy for rechargeable batteries. Curr. Opin. Electrochem. 17: 134-142.

Sokolov, Y., Henderson, G.S., and Wicks, F.J. (2000). Model dependence of AFM simulations in non-contact mode. Surf. Sci. 457: 267-272.

Taeyun, K., Sundaram, G., and Kilho, E. (2019). Atomic force microscopy-based cancer diagnosis by detecting cancerspecific biomolecules and cells. BBA - Rev. Cancer 1871: 367-378.

Tan, B., Zhang, S., and Qiang, Y. (2017). Investigation of the inhibition effect of Montelukast Sodium on the copper corrosion in $0.5 \mathrm{~mol} /$ $\mathrm{L} \mathrm{H}_{2} \mathrm{SO}_{4}$. J. Mol. Liq. 248: 902-910.
Thomé, T., Fouchez, S., and Delalande, S. (2009). Determination of silicone coating Young's modulus using atomic force microscopy. Phys. B Condens. Matter 404: 22-25.

Tianbao, Z., Wenfeng, J., Huilong, W., and Shufen, Z. (2019). Synthesis and localized inhibition behaviour of new triazine-methionine corrosion inhibitor in $1 \mathrm{M} \mathrm{HCl}$ for 2024-T3 aluminium alloy. Mater. Chem. Phys. 237: 121866.

Ting, Y., Shengtao, Z., Li, F., Yujie, Q., Lansi, L., Denglin, F., Yanan, W., Jida, C., Wenpo, L., and Bochuan, T. (2020). Investigation of imidazole derivatives as corrosion inhibitors of copper in sulfuric acid: combination of experimental and theoretical researches. J. Taiwan Inst. Chem. Eng. 106: 118-129.

Tingru, C., Inger, O.W., Ying, J., and Leygraf, C. (2018). The golden alloy Cu-5Zn-5Al-1Sn: a multi-analytical surface characterization. Corros. Sci. 131: 94-103.

Voigtländer, B. (2019). Static atomic force microscopy, atomic force microscopy. Cham: International Publishing, pp. 199-208.

Wuweikai, X., Yanling, T., and Xianping, L. (2020). Dynamic analysis of tapping mode atomic force microscope (AFM) for critical dimension measurement. Precis. Eng. 64: 269-279.

Xiaonan, S., Weihua, Q., Taha, M., and Wen, Z. (2019). Atomic force microscopy - scanning electrochemical microscopy (AFM-SECM) for nanoscale topographical and electrochemical characterization: principles, applications and perspectives. Electrochimica Acta. 332: 135472.

Yakun, Z., Jonathan, D.P., Sirui, L., Raymond, R.U., Leslie, G.B., Christopher, D.T., Jenifer, S.L., Emmanuelle, A.M., and Gerald, S.F. (2020). Localized corrosion at nm-scale hardening precipitates in Al-Cu-Li alloys. Acta Mater. 189: 204-213.

Yang, A.C.M. (1995). Young's moduli of material in polymer deformation zones by an AFM deflection technique. Mater. Chem. Phys. 41: 295-298.

Ying, J., Ming, S., Daobin, M., Xuechong, R., Qingmei, W., and Lei, W. (2012). Investigation of PEG adsorption on copper in $\mathrm{Cu}^{2+}{ }_{\text {-free }}$ solution by SERS and AFM. Electrochim. Acta 78: 459-465.

Yunzhu, S., Liam, C., Nina, B., Peter, K.L., and Bin, Y. (2018). In-situ electrochemical-AFM study of localized corrosion of AlxCoCrFeNi high-entropy alloys in chloride solution. Appl. Surf. Sci. 439: 533-544.

Zhao, W., Song, W., Cheong, L.Z., Wang, D., Li, H., Flemming, B., Huang, F., and Shen, C. (2019). Beyond imaging: applications of atomic force microscopy for the study of Lithium-ion batteries. Ultramicroscopy 204: 34-48. 